\title{
Working Fluid Quantity Effect on Magnetic Field Control of Heat Pipes
}

\author{
F. Cingroš, T. Hron
}

This paper deals with heat pipes controlled by a static magnetic field and with an important side effect - the quantity of working fluid. Heat pipes are able to provide very effective heat transport. Several standard regulation methods are commonly used for this purpose. In previous experiments implemented in our laboratory, we have observed the significant influence of a magnetic field on the heat conductance of the selected heat pipe. A special heat pipe was manufactured for this purpose and pure oxygen was chosen as a working fluid, due to its suitable magnetic properties. The heat pipe operation and the magnetic field control depend on various parameters. This paper is focused on the influence of the quantity of working fluid. Some important results of our experiments are presented and discussed.

Keywords: Heat pipes, magnetic field, variable conductance, oxygen, cryogenic.

\section{Introduction}

A heat pipe is a device that is able to transport heat over long distance with an insignificant temperature drop and with very high efficiency. Heat is transferred by the cycle of a working fluid in a gaseous and liquid state inside a heat pipe, with phase changes - evaporation and condensation. The thermal conductance of a heat pipe is usually more than one hundred times higher than thermal conductance of copper (depending on the type of a heat pipe). Heat pipes are commonly used for cooling and heat transport in electronic devices, technological processes and in many other types of equipment.

In some applications, heat transport controllability is required. Several standard modifications are used for this purpose, e.g. addition of a noncondensable gas into the heat pipe. In previous experiments implemented in our laboratory, we have observed a significant influence of the static magnetic field on the heat transport in the gravitational heat pipe filled with pure oxygen as a working fluid.

Liquid oxygen flowing down a wall under gravity can be captured by a magnetic field. When the magnetic field intensity is high enough, most of the oxygen is trapped. The lower part of the heat pipe is cut off from the working fluid and is apparently inactivated. We have observed that the heat flow is clearly disturbed by the exposure to a static magnetic field with the magnetic induction over $0.4 \mathrm{~T}$. Without magnetic field the working fluid can circulate with no restrictions [1].

The heat pipe operation and the magnetic field control may depend on various parameters, e.g. some of the important properties and the quantity of working fluid (esp. thermal characteristics and the magnetic susceptibility), the construction and the inner structure of the heat pipe (esp. a wick structure), etc. Of course, magnetic field behaviors are also very important for successful heat transfer control.

The influence of the quantity of working fluid in the selected type of heat pipe on the operation and on the magnetic field control has been ascertained in our experiments and will be discussed below. The cryogenic gravitational heat pipe with a special construction was manufactured for this purpose and a testing device was also set up. Oxygen was chosen as a working fluid, due to its suitable magnetic properties. The experimental installation is shown in Fig. 1.

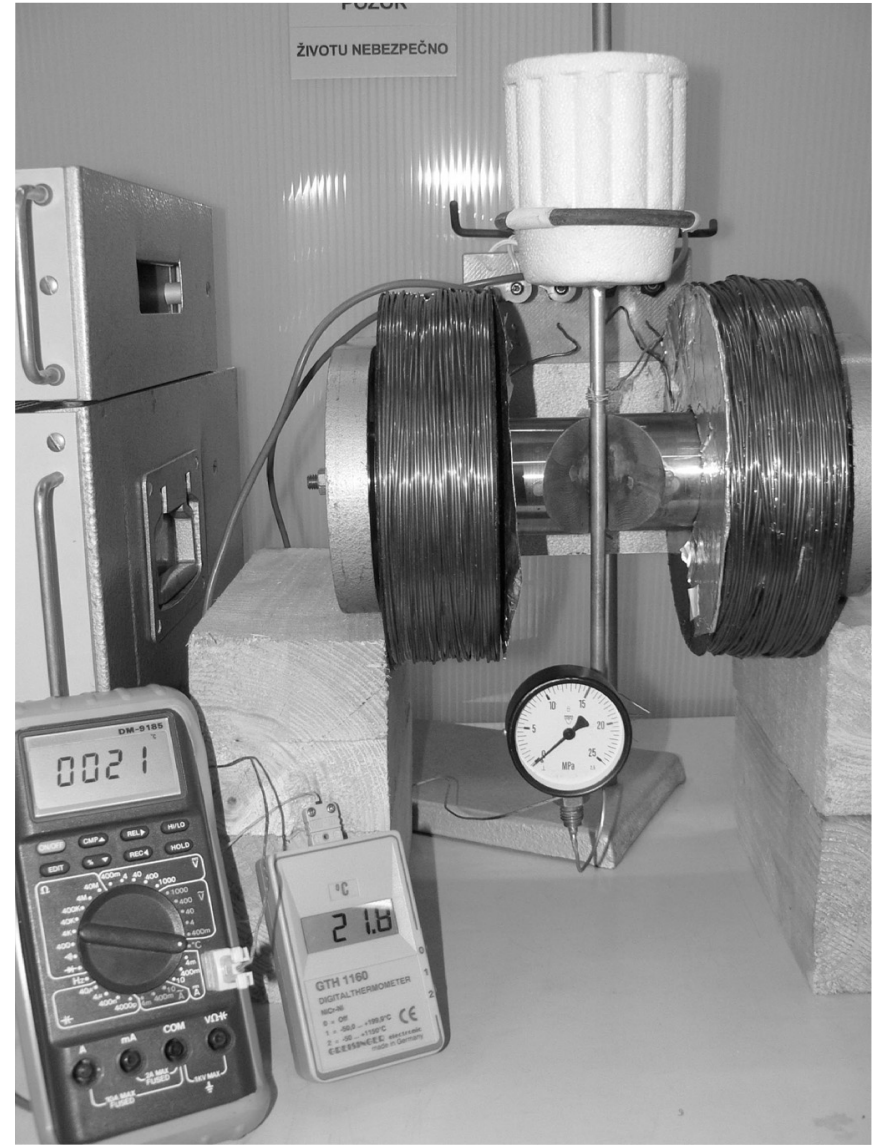

Fig. 1: Experimental installation

\section{Experimental}

A series of identical experiments was performed for several different quantities of working fluid. The influence on the function and on the magnetic field control was observed for the gravitational heat pipe filled with pure oxygen (see Fig. 2). The top part of the heat pipe was chilled by liquid nitrogen and the rest was exposed to room temperature (approx. 297 K). A static magnetic field was situated in the middle. The blocking efficiency of the magnetic field was determined by measuring the temperature changes above and below the magnetic field zone. We used K-type thermocouples calibrated by a Pt-thermometer. 


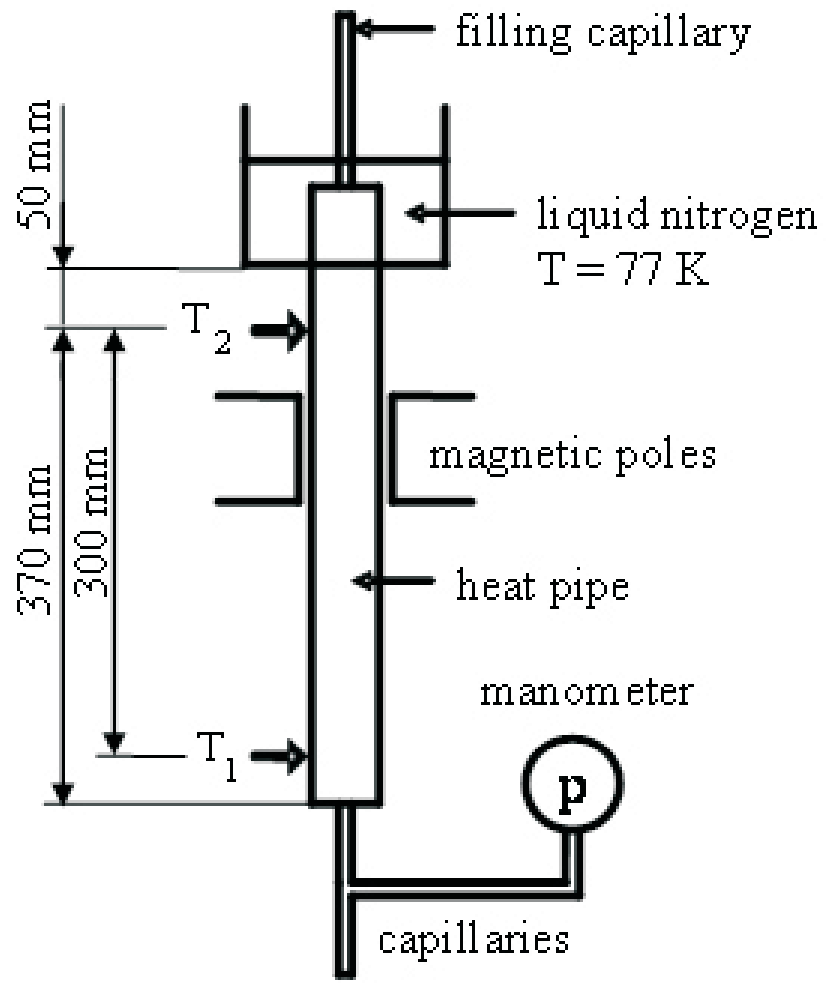

Fig. 2: Schema of the heat pipe system (dimensions are shown in millimeters)

The heat pipe was made from a brassy tube (length $470 \mathrm{~mm}$, outside diameter $8 \mathrm{~mm}$, wall thickness $1 \mathrm{~mm}$ ). The ends of the tube were closed by brassy plugs with a hole through. Copper capillaries (outside diameter $2 \mathrm{~mm}$, wall thickness $0.5 \mathrm{~mm}$ ) were connected through the brassy plugs to both ends of the heat pipe. They connected the heat pipe with a filling device and with a manometer.

The heat pipe was filled with pure oxygen from the pressure vessel through the filling capillary and the filling pressure in the system was measured by the manometer. The relative permeability $\mu_{r}$ of gaseous oxygen is 1.00053 , but for liquid oxygen $\mu_{r}=1.003$ (at boiling point). This is high enough to be possible to capture liquid oxygen by the exposure to a magnetic field.

A static magnetic field was generated by two special $\mathrm{Nd}-\mathrm{Fe}-\mathrm{B}$ permanent magnets (dimensions in millimeters $40 \times 20 \times 10$ ) with a magnetic circuit. The magnetic induction $B$ was $0.6 \mathrm{~T}$ in the middle of the air-gap and the magnetic field was in the active blocking zone approximately homogeneous. The magnetic field could alternatively have been generated by an electromagnet with specially shaped magnetic poles. The magnetic induction $\mathrm{B}$ was adjustable in the range from $0 \mathrm{~T}$ to $1.3 \mathrm{~T}$ in the middle of the air-gap and the gradient $B$ increased to a value of $300 \mathrm{~T} / \mathrm{m}$ at the edges of the poles in such a case.

The experiment was performed for 3 different values of the filling pressure in the heat pipe system - 8.0 MPa, 4.5 MPa, 1.8 MPa (at normal temperature $T=297 \mathrm{~K}$ ). Accordingly, the values of liquid oxygen at liquid nitrogen temperature $\left(T=77 \mathrm{~K}\right.$ ) were $1.33 \mathrm{~cm}^{3}, 0.75 \mathrm{~cm}^{3}, 0.30 \mathrm{~cm}^{3}$ (most of the working fluid is in the liquid state at that moment).

\section{Experimental results}

The functional quality and the magnetic field control of the tested heat pipe were the main parameters observed for the three different quantities of the working fluid. The measured temperature characteristics are illustrated in the three figures, one for each value of the quantity of working fluid. The characteristic $T_{1}$ represents the temperature in the lower part of the heat pipe - below the magnetic field zone, $T_{2}$ represents the temperature in the upper part of the heat pipe between the magnetic field zone and the liquid nitrogen bath (see Fig. 2).

A magnetic field $(B=0.6 \mathrm{~T})$ was active at the beginning of the experiments, then the magnetic field was deactivated for a time period and finally it was activated again till the

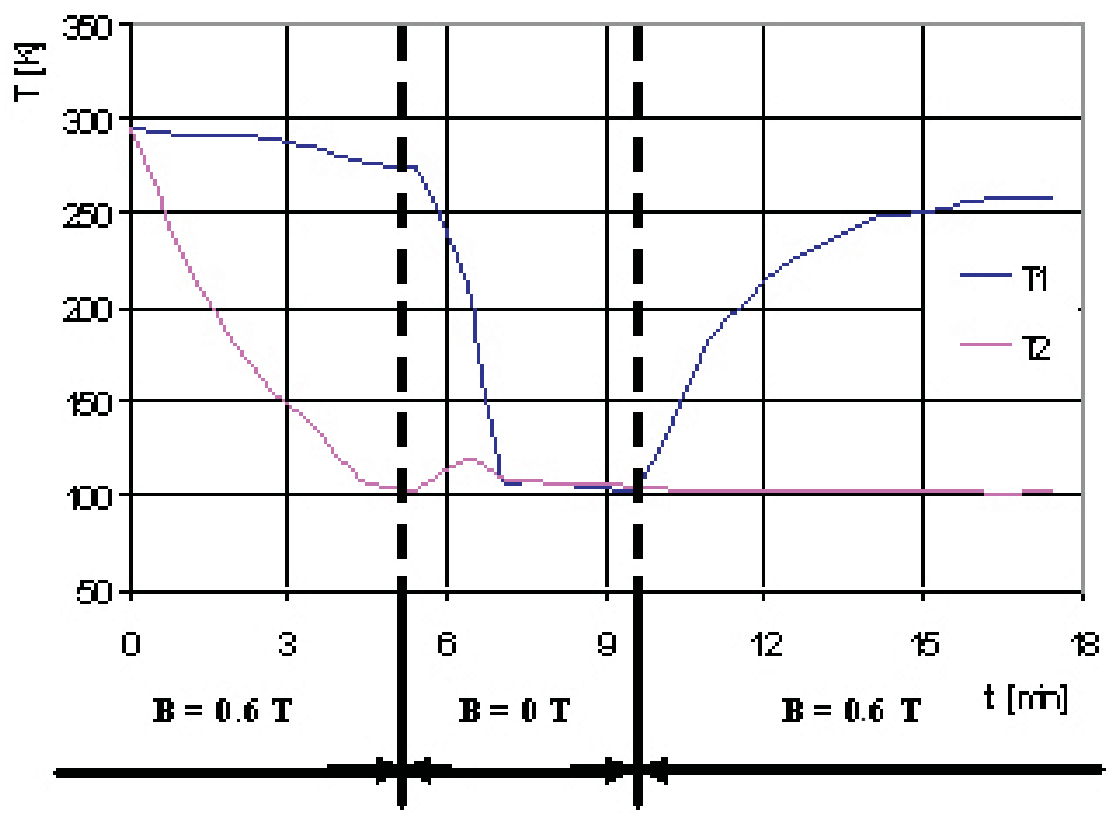

Fig. 3: Temperature characteristics for the heat pipe with filling pressure $8 \mathrm{MPa}$ under discontinuous magnetic field exposure $\mathrm{B}=0.6 \mathrm{~T}$ 


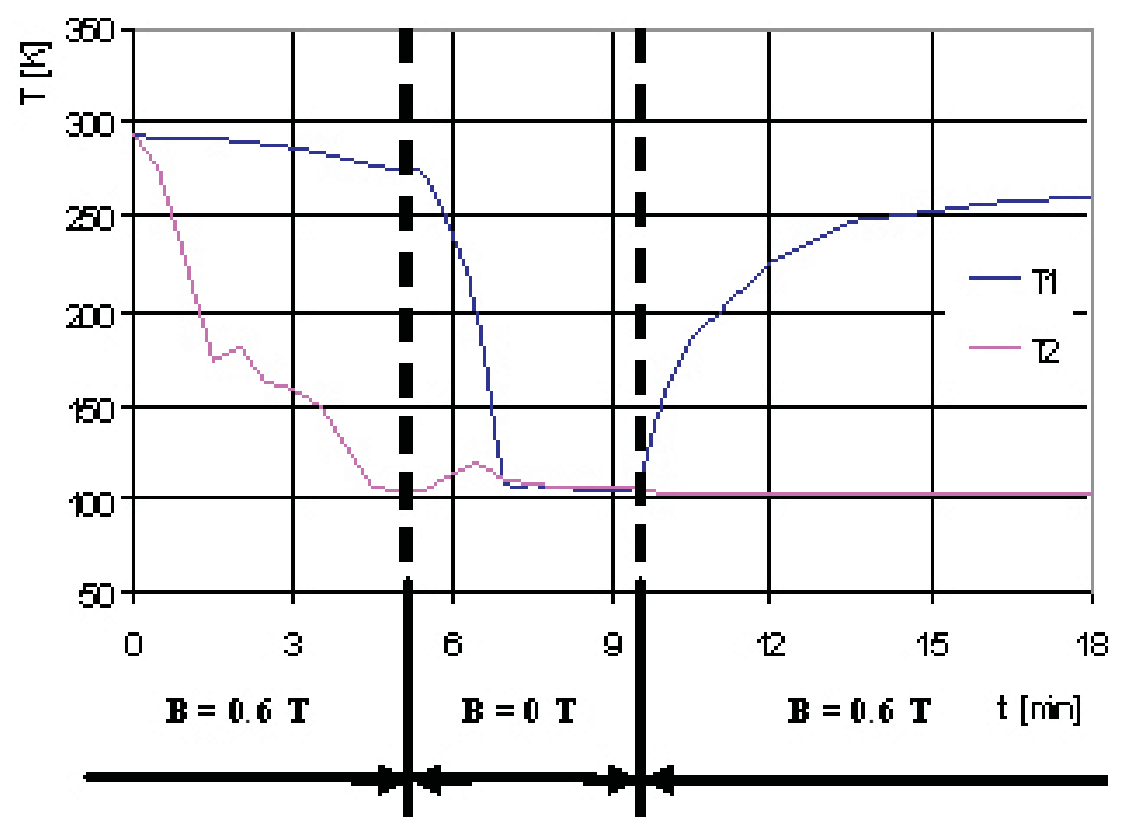

Fig. 4: Temperature characteristics for the heat pipe with filling pressure 4.5 MPa under discontinuous magnetic field exposure $B=0.6 \mathrm{~T}$

end of the experiment. The efficiency of the magnetic field barrier can be determined from the temperature difference $T_{1}-T_{2}$. Without exposure to a magnetic field heat pipe can normally operate and the temperature difference is insignificant. As the disturbing effects increase, the temperature difference also increases.

The heat pipe was able to transport heat in all observed cases and this main function was satisfactory throughout the experiment and with all quantities of working fluid. The heat pipe became almost isothermal in a short time after deactivation of the magnetic field. There is no important difference between the characteristics for filling pressure 8.0 MPa (Fig. 3) and 4.5 MPa (Fig. 4). At $1.8 \mathrm{MPa}$ (Fig. 5) the isothermal temperature was higher. It is obvious that the heat transfer capability was lower in this case and the liquid nitrogen bath was not able to cool the heat pipe as much as before.

Now let us focus on the magnetic field control. The heat transport was clearly disturbed during the exposure to a magnetic field in all observed cases. The magnetic field $(B=0.6 \mathrm{~T})$ was great enough to capture most of the working fluid in the magnetic field zone and the lower part of the heat pipe was cut off and put out of operation. The temperature characteristics for filling pressure 8.0 MPa (Fig. 3) and 4.5 MPa (Fig. 4) are very similar. After deactivation of the

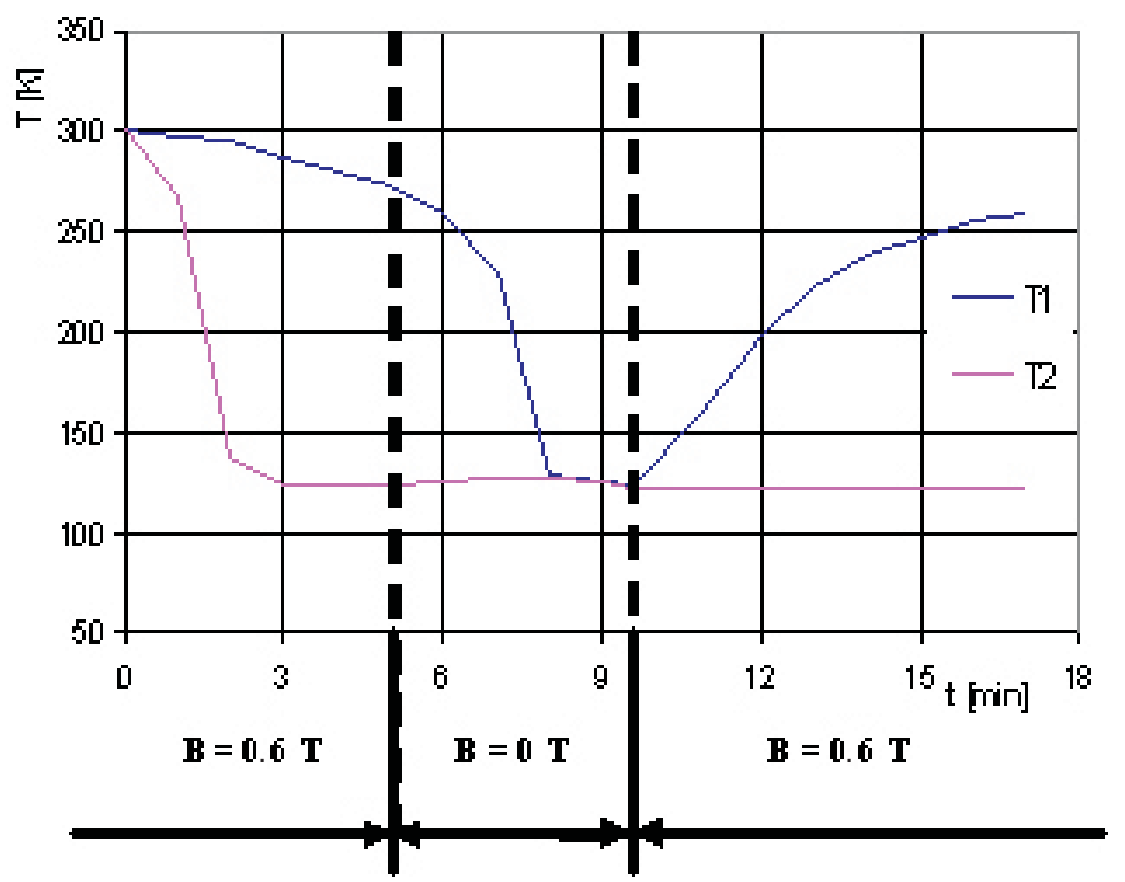

Fig. 5: Temperature characteristics for the heat pipe with filling pressure $1.8 \mathrm{MPa}$ under discontinuous magnetic field exposure $B=0.6 \mathrm{~T}$ 
magnetic field the working cycle inside the heat pipe was refreshed and temperature $T_{1}$ immediately dropped. The temperatures became isothermal in a moment. As soon as the magnetic field was reactivated the temperature difference started to increase rapidly. At filling pressure 1.8 MPa (Fig. 5) the dynamic of these temperature changes was slower than in the previous cases.

The highest temperature difference was achieved at filling pressure 4.5 $\mathrm{MPa}-167 \mathrm{~K}$ just before deactivation of the magnetic field, resp. $159 \mathrm{~K}$ in the time stable state at the end of the measurement. At $8 \mathrm{MPa}$ the difference was slightly lower $-165 \mathrm{~K}$, resp. $156 \mathrm{~K}$. The lowest temperature difference was observed at pressure $1.8 \mathrm{MPa}-155 \mathrm{~K}$, resp. $137 \mathrm{~K}$.

\section{Conclusion}

The influence of the quantity of working fluid on the operation and on the magnetic field control of the mentioned heat pipe has been observed. The cryogenic gravitational heat pipe filled with pure oxygen was specially manufactured and the testing device was set up for this purpose.

The main function of a heat pipe - heat transport - was satisfactory throughout the experiment and with all quantities of working fluid. At filling pressure 1.8 MPa the heat transfer capability was lower than in the case of a greater quantity of working fluid.

The heat transport was clearly disturbed during exposure to the magnetic field exposure in all observed cases. The highest temperature difference under exposure to a magnetic field was achieved at filling pressure 4.5 MPa. At $8 \mathrm{MPa}$ the difference was slightly lower. The lowest temperature difference was observed at pressure 1.8 MPa and the dynamic of the temperature changes was also slower than in the previous cases.

From the set of three quantities of working fluid observed in the experiment, the value for filling pressure $4.5 \mathrm{MPa}$ seems to be optimal. A greater quantity should not make major changes. Reducing the quantity can cause negative effects mentioned above.

\section{Acknowledgments}

The research described in this paper has been supervised by Doc. Ing. J. Kuba, CSc., FEE CTU in Prague and supported by research program no. MSM 6840770012 "Transdisciplinary Research in the Area of Biomedical Engineering II" of the CTU in Prague, sponsored by the Ministry of Education, Youth and Sports of the Czech Republic.

\section{References}

[1] Cingroš, F., Hron, T., Kuba, J.: Magnetic Field Control of Cryogenic Heat Pipes. In Proceedings of the International Conference ISSE 2009. Brno (Czech Republic), 2009.

[2] Kuba, J., Hron, T., Cingroš, F.: Vliv magnetického pole na transport tepla. In Proceedings of the International Conference Diagnostika 2007. Plzeň (Czech Republic), 2007.

[3] Cingroš, F.: Transport tepla tepelnými trubicemi. Master's Thesis at the Dept. of Electrotechnology, FEE CTU in Prague. Prague (Czech Republic), 2007.

[4] Kuba, J.: Ascertaining of the Effects of Magnetic Curtain. In Proceedings of the International Conference Diagnostika 2005. Plzeň (Czech Republic), 2005.

[5] Ueno, S., Iwaki, S., Tazume, K.: Control of Heat Transport in Heat Pipes by Magnetic Field. J. Appl. Phys., Vol. 69 (1991), No. 8, p. 4925-4927.
Filip Cingroš
e-mail: cingrf1@fel.cvut.cz
Tomáš Hron
e-mail: hront1@fel.cvut.cz

Department of Electrotechnology

Czech Technical University in Prague

Faculty of Electrical Engineering

Technická 2

16627 Praha, Czech Republic 